\title{
CHARACTERISATIONS OF THE RIGHT MULTIPLIERS FOR $L_{1}(G, A)$
}

\author{
by MING-KAM CHAN
}

(Received 12th January 1978)

\section{Introduction}

The purpose of this paper is to provide a vector version of the characterisations of the multipliers for $L_{1}(G)$ (see (9), p. 2). This problem was considered by Akinyele (1). However, the Banach algebras involved in that paper are commutative semi-simple Banach algebras and the proof of the main theorem seems to be incomplete. Indeed we give at the end of this paper an example which shows that statements (i) and (ii) of Theorem 3.2 of Akinyele ((1), p. 490) are not equivalent in general.

The plan of this paper is as follows. In Section 2 we collect notation and terminologies which will be used later. In Section 3 several preliminary lemmas will be introduced. In Section 4 the generalised characterisations of the right multipliers for $L_{1}(G, A)$ will be studied.

I should like to thank my research supervisor, Dr A. J. White, for his kind encouragement and valuable suggestions at all times.

\section{Notation and Terminology}

Throughout this paper $A$ will be a Banach algebra with $A^{*}$ and $A^{* *}$ denoting its first and second dual spaces respectively and we shall write $\hat{a}$ for the canonical image of $a \in A$ in $A^{* *}$ and $\hat{A}=\{\hat{a}: a \in A\}$. A net $\left\{u_{\alpha}\right\}$ is a weak approximate identity for $A$ if there is a $M>0$ such that $\left\|u_{\alpha}\right\| \leqslant M$ for all $\alpha$ and $\left\{F\left(a u_{\alpha}-a\right)\right\}$ and $\left\{F\left(u_{\alpha} a-a\right)\right\}$ converge to 0 for each $F \in A^{*}$. We note that ((2), p. 59) the existence of such an identity implies the existence of a norm bounded approximate identity for $A$. In the sequel $A$ is as sumed to have such an identity.

We introduce an Arens product $\times$ in $A^{* *}(5)$ as follows: Let $a, b \in A, f \in A^{*}$ and $F, G \in A^{* *}$. Define $f_{a} \in A^{*}$ by $f_{a}(b)=f(a b)$. Define $f^{F} \in A^{*}$ by $f^{F}(a)=F\left(f_{a}\right)$. Define $f \times G \in A^{* *}$ by $F \times G(f)=F\left(f^{G}\right)$.

A bounded linear operator on $A$ is called a right multiplier on $A$ if $T(a b)=a(T b)$ $(a, b \in A)$. It is shown in $(11$, p. 270) that there is an isometric anti-isomorphism $\Phi$ from the Banach algebra $\mathcal{M}_{r}(A)$ of all right multipliers on $A$ into $A^{* *}$ with the Arens product defined above.

Note that if $a \in A$ the map $R_{a}(b)=b a$ is in $\mathcal{M}_{r}(A)$ and the map $a \rightarrow R_{a}$ is an isometric anti-isomorphism. It is also known that $\Phi\left(\left\{R_{a}: a \in A\right\}\right)=\hat{A}$. We write $A^{(m)}$ for $\Phi\left(\mathcal{M}_{r}(A)\right)$ and we also note that if $A$ has a unit element then $A^{(m)}=\hat{A}$.

Throughout this paper $G$ will be a locally compact group and $\lambda$ a fixed but arbitrary left Haar measure on $G$. The set of all Borel sets in $G$ will be denoted by 
$\mathscr{B}(G)$. The symbol $M(G, A)$ will stand for the space of all $A$-valued regular countably additive measures on $\mathscr{B}(G)$ which have finite variation. Complete details of vector measures can be found in (4) or (5).

$M(G, A)$ is a Banach algebra under the norm $\|\mu\|=|\mu|(G)$ and the convolution defined by $\int_{G} f d \mu^{*} \nu=\int_{G} d \mu(s) \int_{G} f(s t) d \nu(t)\left(f \in C_{0}(G)\right)$ (see (4), p. 162 and (12)). The space of all Bochner-integrable functions from $G$ to $A$ with respect to $\lambda$ will be denoted by $L_{1}(G, A)$. This space actually forms a Banach algebra under the norm defined by $\|f\|_{1}=\int_{G}\|f\|(x) d \lambda(x)$ and the convolution defined by $(f * g)(x)=$ $\int_{G} f(x y) g\left(y^{-1}\right) d \lambda(y)$ (see (8)). If $G$ is abelian and $\mu \in M(G, A)$, the function $\tilde{\mu}: \hat{G} \rightarrow A$ defined by $\tilde{\mu}(\gamma)=\int_{G} \bar{\gamma}(x) d \mu(x)(\gamma \in \hat{G})$ is called the Fourier-Stieltjes transform of $\mu$.

Given two Banach spaces $X$ and $Y$, the projective (resp. injective) tensor product of $X$ and $Y$ will be denoted by $X \hat{\otimes} Y$ (resp. $X \otimes Y$ ) (see (4) or (10)). If $X_{i}, Y_{i}$ $(i=1,2)$ are Banach spaces and $T_{i}: X_{i} \rightarrow Y_{i}(i=1,2)$ are bounded linear operators then the equation $T_{1} \otimes T_{2}\left(x_{1} \otimes x_{2}\right)=T_{1}\left(x_{1}\right) \otimes T_{2}\left(x_{2}\right)$ defines a bounded linear operator from $X_{1} \hat{\otimes} X_{2}$ to $Y_{1} \hat{\otimes} Y_{2}((10)$, p. 37).

\section{Preliminary lemmas}

Lemma 1. Let $\left\{\mu_{\alpha}\right\}_{\alpha \in \Lambda}$ and $\left\{f_{\beta}\right\}_{\beta \in M}$ be respectively bounded approximate identities for $A$ and $L_{1}(G)$. Then $\left\{\mu_{\alpha} f_{\beta}\right\}_{(\alpha, \beta) \in \Lambda \times M}$ is a bounded approximate identity for $L_{1}(G, A)$.

Proof. It follows from the fact that $L_{1}(G, A) \cong L_{1}(G) \hat{\otimes} A((4)$, p. 228).

Lemma 2. $L_{1}(G, A)$ can be embedded in $M(G, A)$ and it is a closed two-sided ideal of $M(G, A)$.

Proof. Define a mapping $F: L_{1}(G, A) \rightarrow M(G, A)$ by $F(f)=\mu_{f}$ where $\mu_{f}(E)=$ $\int_{E} f d \lambda(E \in \mathscr{B}(G)) . F$ is obviously linear. By Dunford-Schwartz ((5), p. 114), $F$ is an isometry. Since $L_{1}(G, A) \cong L_{1}(G) \hat{\otimes} A$, we can find $f_{n}=\Sigma f_{i, n} \otimes a_{i, n}$ and $g_{n}=$ $\Sigma g_{j, n} \otimes b_{j, n}$ such that $f_{n} \rightarrow f$ and $g_{n} \rightarrow g$ in $L_{1}(G) \hat{\otimes} A$. Consequently, we have $\mu_{f * g}=$ $\lim _{n} \mu_{f_{n} * g_{n}}=\lim _{n} \mu_{f_{n}} * \mu_{g_{n}}=\mu_{f} * \mu_{g}$. Hence $L_{1}(G, A)$ can be regarded as a closed subalgebra of $M(G, A)$. To show that it is a right ideal, it is sufficient to show that for any $\nu \in M(G, A), \mu_{a h} * \nu \in L_{1}(G, A)$ for each $a \in A$ and $h \in C_{\infty}(G)$. For any $g \in C_{\infty}(G)$,

$$
\begin{aligned}
\int_{G} g d \mu_{a h} * \nu & =\int_{G} d \mu_{a h}(s) \int_{G} g(s t) d \nu(t) \\
& =\int_{G} d a \mu_{h}(s) \int_{G} g(s t) d \nu() \\
& =\int_{G} d \mu_{h}(s) \int_{G} g(s t) d \omega(t)
\end{aligned}
$$

where $\omega(E)=a \nu(E)(E \in \mathscr{B}(G))$, it is clear that $\omega \in M(G, A)$. Now for each $x^{*} \in A^{*}$, we have, by setting $x^{*} \omega(E)=x^{*}(\omega(E))(E \in \mathscr{B}(G))$,

$$
\begin{aligned}
x^{*} \int_{G} g d \mu_{a h} * \nu & =\int_{G} d \mu_{h}(s) \int_{G} g(s t) d x^{*} \omega(t) \\
& =\int_{G} g d \mu_{h} * x^{*} \omega
\end{aligned}
$$


so $x^{*}\left(\mu_{a h} * \nu\right)=\mu_{h} * x^{*} \omega$. By Hewitt-Ross $\left((6)\right.$, vol. 1, p. 290) we have $\mu_{h} * x^{*} \omega(E)=$ $\int_{E} x^{*} p(t) d \lambda(t)(E \in \mathscr{B}(G))$ where $p(t)=\int_{G} \Delta\left(y^{-1}\right) h\left(x y^{-1}\right) d \omega(y)$ and $p \in L_{1}(G, A)$. Hence $L_{1}(G, A)$ is a right ideal. Similarly we can prove that it is also a left ideal.

Remark: Throughout the rest of this paper, we set

$$
f * \mu=\mu_{f} * \mu \text { and } \overparen{L_{1}(G, A)}=\left\{\tilde{\mu}_{f}: f \in L_{1}(G, A)\right\}
$$

Lemma 3. $M\left(G, A^{* *}\right) \cong\left(C_{0}(G) \otimes A^{*}\right)^{*}$.

Proof. By Diestel-Uhl ((4), p. 162 and p. 169), we have $M\left(G, A^{* *}\right) \cong$ $I\left(C_{0}(G), A^{* *}\right)$ where $I\left(C_{0}(G), A^{* *}\right)$ denotes the space of all integral operators from $C_{0}(G)$ to $A^{* *}$. The result then follows from Diestel-Uhl ((4), p. 237).

Remark: For each $T \in I\left(C_{0}(G), A^{* *}\right)$, its image under the isomorphism in $\left(C_{0}(G) \otimes A^{*}\right)^{*}$ is the functional whose value at $f \otimes x^{*}$ is $T(f)\left(x^{*}\right)$.

\section{Main Theorem}

The main results of the right multipliers on $L_{1}(G, A)$ are contained in the following theorem. A classical analogue of this result can be found in Larsen ((9), p. 2).

Theorem. Let $G$ be a locally compact group and let $A$ be a Banach algebra with a weak approximate identity. If $T: L_{1}(G, A) \rightarrow L_{1}(G, A)$ is a bounded linear operator, then the following assertions are equivalent:

(i) $T(f * g)=f * T g$ for each $f, g \in L_{1}(G, A)$.

(ii) There exists a unique measure $\mu \in M\left(G, A^{(m)}\right)$ such that $T f=f * \mu$ for each $f \in L_{1}(G, \hat{A})$.

(iii) $T \circ\left(\lambda_{a} \hat{\otimes} \tau_{s}\right)=\left(\lambda_{a} \hat{\otimes} \tau_{s}\right) \circ T$ for each $a \in A$ and $s \in G$ where $\lambda_{a}$ is the operator on $A$ given by $\lambda_{a}(b)=a b$ and $\tau_{s}$ is the translation operator on $L_{1}(G)$ given by $\tau_{s}(f)(t)=f\left(s^{-1} t\right)$.

Furthermore, if $G$ is abelian, the above statements are equivalent to the following statements:

(iv) There exists a unique measure $\mu \in M\left(G, A^{(m)}\right)$ such that $\widetilde{T f}=\tilde{f} \tilde{\mu}$ for each $f \in L_{1}(G, \hat{A})$.

(v) There exists a unique function $\phi: \hat{G} \rightarrow A^{(m)}$ such that $\tilde{f} \phi \in L_{1}(G, \hat{A})$ for each $f \in L_{1}(G, \hat{A})$. Furthermore the function $\phi$ is related to $T$ by the equation $\overline{T f}(\gamma)=$ $\tilde{f}(\gamma) x \phi(\gamma)(\gamma \in \hat{G})$.

Proof. (i) $\Rightarrow$ (ii). Since $A$ has bounded approximate identity, by Lemma 1, $L_{1}(G, A)$ has bounded approximate identity $\left\{f_{\beta}\right\}$. Clearly the set $\left\{T f_{\beta}\right\} \subset M\left(G, A^{* *}\right)$ is bounded. By Lemma 3 and Alaoglu's Theorem, we obtain a $\mu \in M\left(G, A^{* *}\right)$ such that

$$
T f_{\beta}(f) x^{*} \rightarrow \mu(f) x^{*} \text { for each } f \in C_{0}(G) \text { and } x^{*} \in A^{*} \ldots(*) .
$$

Let $a \in A, f, g \in C_{\infty}(G)$. Then for each $x^{*} \in A^{*}$, we have 


$$
\begin{aligned}
\left|x^{*} \int_{G} f d\left(T\left(a g * f_{\beta}\right)\right)-\int_{G} f d(\hat{a} g * \mu)\left(x^{*}\right)\right|= & \left|x * \int_{G} f d\left(a g * T f_{\beta}\right)-\int_{G} f d(\hat{a} g * \mu)\left(x^{*}\right)\right| \\
= & \mid \int_{G} g(s)\left[\int_{G} f(s t) d x^{*}\left(a T f_{\beta}\right)(t)\right] d \lambda(s) \\
& -\int_{G} g(s)\left[\int_{G} f(s t) d(\hat{a x} \mu)(t)\right] d \lambda(s)\left(x^{*}\right) \mid \\
= & \mid x^{*} \int_{G} d a T f_{\beta}(t) \int_{G} f(s t) g(s) d \lambda(s) \\
& -\int_{G} d(\hat{a} x \mu)(t) \int_{G} f(s t) g(s) d \lambda(s)\left(x^{*}\right) \mid \\
= & \left|x^{*} \int_{G} h(t) \operatorname{daTf}(t)-\hat{a x} \mu(h)\left(x^{*}\right)\right|
\end{aligned}
$$

where $h(t)=\int_{G} f(s t) g(s) d \lambda(s) \in C_{\infty}(G)$ and $(\hat{a x} \mu)(E)=\hat{a} x \mu(E)(E \in \mathscr{B}(G))$.

If we define $y^{*}(b)=x^{*}(a b)$ for each $b \in A$, then $Y^{*} \in A^{*}$. By definition of Arens product, we have

$$
\left|x^{*} \int_{G} h(t) \operatorname{daTf} f_{\beta}(t)-\hat{a x} \mu(h)\left(x^{*}\right)\right|=\left|T f_{\beta}(h)^{\wedge} y^{*}-\mu(h) y^{*}\right|
$$

Hence by (*), we obtain

$$
\begin{aligned}
\mid x^{*} \int_{G} f d\left(T\left(a g * f_{\beta}\right)\right) & -\int_{G} f d(\hat{a} g * \mu)\left(x^{*}\right) \mid \\
& =\left|T f_{\beta}(h)^{\wedge} y^{*}-\mu(h) y^{*}\right| \rightarrow 0
\end{aligned}
$$

Moreover

$$
\left|x^{*} T\left(a g * f_{\beta}\right)(f)-T a g(f)^{\wedge} x^{*}\right| \leqslant\left\|x^{*}\right\|\|T\|\|f\|_{\infty}\left\|a g * f_{\beta}-a g\right\|_{1} \rightarrow 0
$$

Thus we have

$$
\int_{G} f d(\hat{a} g * \mu)\left(x^{*}\right)=\operatorname{Tag}(f)^{\wedge} x^{*} \text { for each } x^{*} \in A^{*}
$$

Hence

$$
\int_{G} f d(\hat{a} g * \mu)=\operatorname{Tag}(f)^{\wedge} \quad\left(f, g \in C_{\infty}(G)\right) .
$$

This implies $\hat{a} g * \mu=$ Tag $\left(g \in C_{\infty}(G)\right)$.

Since $C_{\infty}(G) \hat{\otimes} A \cong L_{1}(G, A)$, we have

$$
f * \mu=T f \quad \text { for each } f \in L_{1}(G, \hat{A}) .
$$

Since for each $a \in A, f, g \in C_{\infty}(G)$

$$
\hat{a} g * \mu(f)=\int_{G} h(s) \operatorname{dax} \mu(s) \in \hat{A} \quad \text { and } \quad h \in C_{\infty}(G) * C_{\infty}(G)
$$

which is dense in $C_{0}(G)$ (see (6), vol 2. p. 283), by Tomiuk ((11), p. 271) and 
Diestel-Uhl ((4), p. 153) we can conclude that $\mu \in M\left(G, A^{(m)}\right)$ and is unique.

(ii) $\Rightarrow$ (iii) Since for each $b \in A$ and $f \in L_{1}(G)\left(\lambda_{a} \otimes \tau_{s}\right)(b \otimes f)=a b \tau_{s} f$, it follows immediately that for each $f \in L_{1}(G, A)\left(\lambda_{a} \hat{\otimes} \tau_{s}\right)(f)=a \tau_{s} f$ where $\tau_{s}$ is regarded as a translation operator on $L_{1}(G, A)$ which is identified with $L_{1}(G) \hat{\otimes} A$. Moreover given any $b \in A$ and $g \in L_{1}(G)$, for each $x^{*} \in A^{*}$, we have, by Theorem 20.9 of HewittRoss ((6), p. 290), $x^{*}\left(\left(b \tau_{s} g\right) * \mu\right)=\left(\tau_{s} g\right) * x *(b \mu)=\tau_{s}\left(g * x^{*}(b \mu)\right)$. So $x^{*}\left(\left(b \tau_{s} g\right) * \mu\right)=$ $\tau_{s}\left(x^{*}(b g * \mu)\right)=x^{*}\left(\tau_{s}(b g * \mu)\right)\left(x^{*} \in A^{*}\right)$. This implies $\left(b \tau_{s} g\right) * \mu=\tau_{s}(b g * \mu)$. Hence for each $b \in A$ and $g \in L_{1}(G)$,

$$
\begin{aligned}
T \circ\left(\lambda_{a} \hat{\otimes} \tau_{s}\right)(b \otimes g) & =T\left(a b \tau_{s} g\right)=a b \tau_{s} g * \mu=a \tau_{s}(b g * \mu) \\
& =\left(\lambda_{a} \hat{\otimes} \tau_{s}\right) \circ T(b g) .
\end{aligned}
$$

(iii) $\Rightarrow$ (i) For each $a \in A$ and $x^{*} \in A^{*}$, define a mapping $f \mapsto x^{*} T(a f)$ from $L_{1}(G)$ to $L_{1}(G)$. Let $\left\{\mu_{\alpha}\right\}$ be a bounded approximate identity for $A$. Then $x^{*} T\left(u_{\alpha} a f\right) \rightarrow x^{*} T(a f)$ and $x^{*}\left(u_{\alpha} T(a f)\right) \rightarrow x^{*} T(a f)$ in $L_{1}(G)$. Consequently,

$$
\begin{aligned}
x^{*}\left(T \circ\left(\lambda_{u_{a}} \hat{\otimes} \tau_{s}\right)(a f)\right) & =x^{*}\left(u_{\alpha} \tau_{s} T(a f)\right) \\
& =\tau_{s}\left(x^{*} u_{\alpha} T(a f)\right) \rightarrow \tau_{s}\left(x^{*} T(a f)\right)
\end{aligned}
$$

and $\quad x^{*}\left(T \circ\left(\lambda_{u_{\alpha}} \hat{\otimes} \tau_{s}\right)(a f)\right)=x^{*} T\left(u_{\alpha} a \tau_{s} f\right) \rightarrow x^{*} T\left(a \tau_{s} f\right)$. By Brainerd-Edwards ((3), p. 303) the mapping defined above is a right multiplier of $L_{1}(G)$. Hence for each $a, b \in A$ and $f, g \in L_{1}(G)$,

$$
\begin{aligned}
x^{*} T(a f * b g) & =x * T(a b f * g)=f * x * T(a b g) \\
& =f * x *\left(T \circ\left(\lambda_{a} \hat{\otimes} T_{e}\right)(b g)\right) \\
& =f * x^{*}(a T(b g)) \\
& =x *(a f * T(b g)) \quad\left(x^{*} \in A^{*}\right) .
\end{aligned}
$$

Therefore $T(a f * b g)=a f * T(b g)$. And (i) follows immediately.

(ii) $\Rightarrow$ (iv) If $\mu \in M\left(G, A^{(m)}\right)$, by Lemma 2 we see that $\hat{a} g * \mu \in L_{1}\left(G, A^{* *}\right)$ and by Lemma 5.1 of Tomiuk we have $\hat{a} g * \mu \in L_{\mathrm{l}}(G, \hat{A})$ for each $a \in A$ and $g \in C_{\infty}(G)$. Hence $f * \mu \in L_{1}(G, \hat{A})$ for each $f \in L_{1}(G, \hat{A})$. Then we can define a mapping $T: L_{1}(G, \hat{A}) \rightarrow L_{1}(G, \hat{A})$ by the equation $T f=f * \mu$. This mapping is obviously a right multiplier of $L_{1}(G, A)$ and (iv) follows from the definition of Fourier-Stieltjes transfrom and White ((12), p. 131).

(iv) $\Rightarrow$ (v) Trivial.

(v) $\Rightarrow$ (i) Define $T: L_{1}(G, \hat{A}) \rightarrow L_{1}(G, \hat{A})$ by the equation $(\widetilde{T f})(\gamma)=\tilde{f}(\gamma) \times \phi(\gamma)$ $(\gamma \in \hat{G})$. By uniqueness of Fourier-Stieltjes transform this mapping is well-defined and linear. Let $f, g \in L_{1}(G, \hat{A})$, then

So $T(f * g)=f * T g$.

$$
\begin{aligned}
T \widetilde{(f * g)}(\gamma) & =\widetilde{(f * g)}(\gamma) \times \phi(\gamma)=\hat{f}(\gamma) \times(\tilde{g}(\gamma) \times \phi(\gamma)) \\
& =\tilde{f}(\gamma) \times(\widetilde{T g})(\gamma)=(\widetilde{f * T g})(\gamma) .
\end{aligned}
$$

Since $L_{1}(G, A)$ has bounded approximate identity, by Johnson ((7), p.640) $T$ is continuous. Hence $T$ is a right multiplier.

Corollary. Let $\mathcal{M}\left(L_{1}(G, A)\right)$ be the space of all right multipliers on $L_{1}(G, A)$. Then $\mathcal{M}\left(L_{1}(G, A)\right)$ is topologically isomorphic to $M\left(G, A^{(m)}\right)$. 
Proof. By the proof of the main theorem, we obtain a mapping $\mu \mapsto T$ from $M\left(G, A^{(m)}\right)$ onto $\mathcal{M}\left(L_{1}(G, A)\right)$ which is linear, one-to-one and bounded. Hence the result follows from the inversion theorem.

\section{Remarks.}

(1) It is in general not sufficient to characterise those right multipliers on $L_{1}(G, A)$ by the following condition:

$$
\tau_{s} \circ T(f)=T \circ \tau_{s}(f) \text { for each } s \in G \text { and } f \in L_{1}(G, A) .
$$

This can be justified by the following example.

Example. Choose a bounded operator $T$ on $A$ which is not a right multiplier on $A$ and let $e$ be the unit element of $G$. Then the operator $T \hat{\otimes} \tau_{e}$ commutes with $\tau_{s}$ for any $s \in G$. However it does not commute with $\lambda_{a} \hat{\otimes} \tau_{s}$ in general because right multipliers on $A$ are characterised by the following condition: $T$ is a right multiplier on $A$ if and only if $T \circ \lambda_{a}=\lambda_{a} \circ T$ for each $a \in A$. So it is not a right multiplier on $L_{1}(G, A)$.

(2) If $A$ has a unit element, then $\mu$ can be regarded as an $A$-valued measure.

\section{REFERENCES}

(1) O. Akinyele, A multiplier problem, Atti. Accad. naz. Lincei, Ser., Rend., Cl. Sci. fis. mat. natur. 57 (1974) 487-490 (1975).

(2) F. F. BONSALl and J. DunCAN, Complete normed algebras (Springer, Berlin, 1973).

(3) B. BRAINERD and R. E. EDWARDS, Linear operators which commute with translations, I. J. Austral. Math. Soc. 6 (1966), 289-327.

(4) J. Diestel and J. J. UHL, Jr., Vector measures (American mathematical society, Math. surveys, number 15,1977 ).

(5) N. DUNFORD and J. SCHWARTZ, Linear operators, vol. 1 (Interscience New York, 1958).

(6) E. HEwITT and K. A. Ross, Abstract harmonic analysis (Springer, Berlin: vol. 1, 1963, vol. 2, 1970).

(7) B. E. Johnson, Continuity of centralisers on Banach algebras. J. London Math. Soc. 41 (1966), 639-640.

(8) G. P. JOHNSON, Spaces of functions with values in a Banach algebra, Trans. Amer. Math. Soc. 92 (1959), 411-429.

(9) R. LARSEN, An introduction to the theory of multipliers (Springer, Berlin, 1971).

(10) R. Schatten, A theory of cross-spaces (Princeton University Press, 1950).

(11) B. J. TомIUK, Multipliers on Banach algebras, Studia Math. 54 (1976), 267-283.

(12) A. J. WhITE, Convolution of vector measures, Proc. Royal Soc. of Edinburgh 73A (1975), 117-135.

\section{DEPARTMENT OF MATHEMATICS \\ UNIVERSITY OF ABERDEEN \\ ABERDEEN AB9 2TY}

